\title{
ESTUDO DO EQUILÍBRIO LÍQUIDO-LÍQUIDO PARA A OTIMIZAÇÃO DO PROCESSO DE PURIFICAÇÃO DE BIODIESEL METÍLICO DE BABAÇU
}

\author{
R. S. PINHEIRO ${ }^{1}$, B. A. QUEIROZ ${ }^{1}$, F. M. R. MESQUITA, H. B. de SANT'ANA ${ }^{1}$ e R. S. \\ de SANTIAGO-AGUIAR ${ }^{1}$
}

${ }^{1}$ Universidade Federal do Ceará, Departamento de Engenharia Química e-mail para contato: rspinheiro3@gmail.com

\begin{abstract}
RESUMO - No processo de purificação do biodiesel, a lavagem da fase éster é de extrema importância, visto que há excessivo consumo de água. Nesta etapa, álcool em excesso é removido. O estudo do equilíbrio líquido-líquido (ELL) de dados composicionais para a lavagem de biodiesel pode fornecer meios para o desenvolvimento de equipamentos e a otimização dos processos de extração. $\mathrm{O}$ principal objetivo deste trabalho foi determinar dados de ELL para o sistema ternário biodiesel de babaçu + metanol + água, a $20{ }^{\circ} \mathrm{C}$ e $60{ }^{\circ} \mathrm{C}$ e pressão atmosférica. A determinação experimental foi realizada por intermédio das técnicas titulométrica e densimétrica. A consistência termodinâmica dos dados foi verificada pela correlação de Othmer-Tobias. Observou-se, através da análise gráfica, influência negligenciável da temperatura para os sistemas estudados. Os dados de ELL foram correlacionados pelos seguintes modelos para coeficiente de atividade: NRTL, UNIQUAC e UNIFAC, com desvio médio global de $0,88 \% ; 0,80 \%$ e 3,96 $\%$, respectivamente.
\end{abstract}

\section{INTRODUÇÃO}

O petróleo é a principal força motriz utilizada nos motores de combustão interna dos sistemas veiculares. No entanto, apresenta uma série de características negativas, como sua origem não renovável. Por esta razão, nas duas últimas décadas, tem sido crescente o número de pesquisas com fontes de energias alternativas que venham a reduzir a grande dependência dos países com relação ao petróleo. Dentre estas alternativas, o uso de biodiesel tem se apresentado como uma bastante promissora, quer por suas características físico-químicas, quer nos âmbitos ambiental e econômico, já sendo uma realidade em vários países tais como Alemanha e Brasil (Knothe et al., 2006). Além disso, pode ser utilizado sem modificações mecânicas nos motores dos veículos (Ferrari et al., 2005).

A matéria-prima para a produção de biodiesel é proveniente, em grande parte, de óleos vegetais (soja, girassol, milho, algodão, babaçu, dentre outros) e gorduras animais. As matériasprimas devem ser compostas prioritariamente por triglicerídeos que, após a reação alcoólica, são convertidos em diferentes ácidos graxos (Knothe et al., 2006). Neste trabalho, a escolha do babaçu como matéria-prima deu-se por características geoeconômicas e tipicidade no nordeste brasileiro, cujo potencial econômico ainda é pouco explorado (Lima et al., 2007).

A reação de transesterificação com metanol, por via catalítica homogênea, é o processo mais simples e comumente utilizado para a conversão de óleos e gorduras em ésteres de 
interesse. Após a reação, o biodiesel é separado por decantação do glicerol e em seguida a massa reacional remanescente passa por lavagens com água. A etapa de lavagem retira álcool em excesso, catalisador e sabão. Segundo De Boni et al. (2007), na indústria, o processo de lavagem requer cerca de $3 \mathrm{~L}$ de água para a purificação de $1 \mathrm{~L}$ de biodiesel.

Neste contexto, o conhecimento das relações de equilíbrio líquido-líquido envolvidas na produção deste biodiesel é essencial para o melhor entendimento do comportamento do sistema. Estes dados são essenciais para realização de simulações computacionais e no ajuste dos processos nas plantas de extração. Adicionalmente, esses dados experimentais foram correlacionados com os modelos termodinâmicos NRTL, UNIQUAQ e UNIFAC.

\section{MATERIAIS E MÉTODOS}

Os dados experimentais foram determinados em células de equilíbrio encamisadas que seguem as especificações propostas por Stragevitch (1997). Os experimentos foram realizados as temperaturas de $20^{\circ} \mathrm{C}$ e $60{ }^{\circ} \mathrm{C}$, controladas através de banho termostatizado, e a pressão atmosférica.

Para a construção das curvas, binodal e de calibração, partiu-se de uma mistura binária de composição conhecida e foi titulado um terceiro componente sob forte agitação até o aparecimento e permanência de turbidez. As duas fases em estudo foram: Fase I (rica em biodiesel): construída a partir de misturas binárias de biodiesel e metanol, tilulando-se com água. Fase II (rica em água): construída a partir de misturas binárias de água e metanol, tilulando-se com biodiesel. Este processo foi repetido para diferentes composições de misturas binárias.

Os dados de densidade em função da fração mássica, foram ajustados por meio de uma equação polinomial de segundo grau (Equação 1), onde $a, b$ e $c$ representam os parâmetros do ajuste polinomial, $\mathrm{x}$ as frações mássicas e $\rho$ a densidade.

$$
\rho=a x^{2}+b x+c
$$

De conhecimento da região heterogênea, limitada pela curva binodal, foram estimados valores de composição global dentro desta curva e pesados nas células de equilíbrio. As misturas foram agitadas por $3 \mathrm{~h}$ e permaneceram em repouso por $14 \mathrm{~h}$, onde foi observado a formação de uma interface bem definida entre as duas fases. Foi medida a densidade das duas fases e estas foram convertidas em fração mássica através das curvas de calibração.

\section{MODELAGEM TERMODINÂMICA}

No desenvolvimento da modelagem foi utilizado o programa Fortran TML-LLE 2.0, (Stragevitch; D’Ávila, 1997), implementado com modelos NRTL (Renon e Prausnitz, 1968), UNIQUAC (Abrams e Prausnitz, 1975) e UNIFAC (Fredenslund et al., 1977). O procedimento é baseado no método Simplex proposto por Nelder e Mead (1965), e consiste na minimização de uma função objetivo (Equação 2) baseada na concentração dos componentes, definida como: 


$$
F . O=\sum_{k}^{D} \sum_{j}^{M} \sum_{i}^{N-1}\left(x_{i j k}^{I, \exp }-x_{i j k}^{I, \text { calc }}\right)^{2}+\left(x_{i j k}^{I I, \exp }-x_{i j k}^{I I, \text { calc }}\right)^{2}
$$

onde, $\mathrm{D}$ é o número de conjunto de dados, $\mathrm{N}$ e $\mathrm{M}$ são o número de componentes e linhas de amarração em cada conjunto de dados, respectivamente; os sobrescritos I e II referem-se às duas fases líquidas em equilíbrio, enquanto os sobrescritos 'exp' e 'calc' referem-se aos valores de concentração experimentais e calculados na fase líquida.

O desvio médio quadrático foi calculado comparando as composições experimentais e as calculadas, de cada componente, em cada uma das duas fases, através da Equação 3:

$$
\delta_{\mathrm{x}}=100 \sqrt{\frac{\sum_{\mathrm{i}}^{\mathrm{M}} \sum_{\mathrm{j}}^{\mathrm{N}}\left(\mathrm{x}_{\mathrm{ij}}^{\mathrm{I}, \text { exp }}-\mathrm{x}_{\mathrm{ij}}^{\mathrm{I}, \mathrm{calc}}\right)^{2}+\left(\mathrm{x}_{\mathrm{ij}}^{\mathrm{II}, \mathrm{exp}}-\mathrm{x}_{\mathrm{ij}}^{\mathrm{II}, \mathrm{calc}}\right)^{2}}{2 \mathrm{MN}}}
$$

\section{RESULTADOS E DISCUSSÃO}

A metodologia adotada neste trabalho (titulometria e densimetria), foi previamente certificada pela reprodução dos dados de Zhang e Hill (1991). Maiores detalhes podem ser consultados em Pinheiro et al. (2014).

Na Tabela 1 são reportadas dados de fração mássica e densidade utilizados na construção da curva binodal e nas curvas de calibração. Pode-se observar que para os sistemas estudados, em ambas as temperaturas, houve um aumento na densidade com o aumento da fração mássica da água. As composições das misturas globais e das fases em equilíbrio são apresentadas na Tabela 2.

Tabela 1 - Dados de densidade e composição das curvas de calibração para o sistema Biodiesel de babaçu (1)

\begin{tabular}{|c|c|c|c|c|c|}
\hline \multicolumn{3}{|c|}{$\begin{array}{c}\text { Biodiesel de babaçu (1) + metanol (2) + água (3) } \\
20{ }^{\circ} \mathrm{C}\end{array}$} & \multicolumn{3}{|c|}{$\begin{array}{l}\text { Biodiesel de babaçu (1) + metanol (2) + água (3) a } \\
\qquad 60^{\circ} \mathrm{C}\end{array}$} \\
\hline $\mathrm{w}_{1}$ & $\mathrm{~W}_{2}$ & Densidade $\left(\mathrm{g} . \mathrm{cm}^{-3}\right)$ & $\mathrm{w}_{1}$ & $\mathrm{~W}_{2}$ & Densidade $\left(\mathrm{g} . \mathrm{cm}^{-3}\right)$ \\
\hline 0,0642 & 0,8983 & 0,8066 & 0,1196 & 0,8308 & 0,7794 \\
\hline 0,1305 & 0,8376 & 0,8070 & 0,2390 & 0,7138 & 0,7883 \\
\hline 0,3598 & 0,6011 & 0,8261 & 0,3558 & 0,5947 & 0,7996 \\
\hline 0,6121 & 0,3676 & 0,8466 & 0,4778 & 0,4781 & 0,8088 \\
\hline 0,7370 & 0,2496 & 0,8556 & 0,6043 & 0,3622 & 0,8192 \\
\hline 0,8661 & 0,1241 & 0,8660 & 0,7313 & 0,2433 & 0,8290 \\
\hline 0,9249 & 0,0697 & 0,8709 & 0,8436 & 0,1195 & 0,8390 \\
\hline 0,0131 & 0,7440 & 0,8588 & 0,9604 & 0,0103 & 0,8467 \\
\hline 0,0062 & 0,4935 & 0,9161 & 0,0290 & 0,8693 & 0,7865 \\
\hline 0,0063 & 0,2492 & 0,9586 & 0,0108 & 0,7442 & 0,8253 \\
\hline 0,0023 & 0,2058 & 0,9656 & 0,0050 & 0,6228 & 0,8568 \\
\hline 0,0026 & 0,0329 & 0,9923 & 0,0029 & 0,4953 & 0,8896 \\
\hline 0,0023 & 0,0100 & 0,9962 & 0,0051 & 0,3738 & 0,9128 \\
\hline- & - & - & 0,0046 & 0,2494 & 0,9373 \\
\hline- & - & - & 0,0041 & 0,1244 & 0,9601 \\
\hline- & - & - & 0,0157 & 0,0101 & 0,9794 \\
\hline
\end{tabular}
+ metanol (2) + água (3) a 20 e $60{ }^{\circ} \mathrm{C}$ 
Tabela 2 - Dados de fração mássica das linhas de amarração para o sistema Biodiesel de babaçu (1) + metanol (2) + água (3) a $20{ }^{\circ} \mathrm{C}$ e $60{ }^{\circ} \mathrm{C}$

\begin{tabular}{|c|c|c|c|c|c|c|c|c|}
\hline \multicolumn{3}{|c|}{ Composição global } & \multicolumn{3}{|c|}{ Fase rica em biodiesel } & \multicolumn{3}{|c|}{ Fase rica em água } \\
\hline $\mathrm{w}_{1}$ & $\mathrm{~W}_{2}$ & $\mathrm{w}_{3}$ & $\mathrm{w}_{1}$ & $\mathrm{w}_{2}$ & $\mathrm{w}_{3}$ & $\mathrm{w}_{1}$ & $\mathrm{w}_{2}$ & $\mathrm{~W}_{3}$ \\
\hline \multicolumn{9}{|c|}{ Biodiesel de babaçu + metanol + água a $20^{\circ} \mathrm{C}$} \\
\hline 0,4974 & 0,0924 & 0,4100 & 0,9848 & 0,0149 & 0,0002 & 0,0031 & 0,1747 & 0,822 \\
\hline 0,5010 & 0,1640 & 0,3350 & 0,9777 & 0,0215 & 0,0008 & 0,0045 & 0,3128 & 0,683 \\
\hline 0,5009 & 0,2380 & 0,2601 & 0,9672 & 0,0313 & 0,0020 & 0,0067 & 0,4555 & 0,538 \\
\hline 0,5034 & 0,3120 & 0,1850 & 0,9519 & 0,0455 & 0,0030 & 0,0096 & 0,6069 & 0,384 \\
\hline 0,4986 & 0,3801 & 0,1210 & 0,9258 & 0,0698 & 0,0040 & 0,0125 & 0,7383 & 0,249 \\
\hline 0,5008 & 0,4002 & 0,0990 & 0,9105 & 0,0840 & 0,0060 & 0,0136 & 0,7828 & 0,204 \\
\hline \multicolumn{9}{|c|}{ Biodiesel de babaçu + metanol + água a $60^{\circ} \mathrm{C}$} \\
\hline 0,4974 & 0,0924 & 0,4100 & 0,9523 & 0,0244 & 0,0230 & 0,0037 & 0,1815 & 0,8150 \\
\hline 0,5010 & 0,164 & 0,3350 & 0,9392 & 0,0371 & 0,0240 & 0,0004 & 0,3229 & 0,6770 \\
\hline 0,5009 & 0,238 & 0,2610 & 0,9184 & 0,0571 & 0,0240 & 0,0025 & 0,4784 & 0,5190 \\
\hline 0,5034 & 0,312 & 0,1850 & 0,8913 & 0,0833 & 0,0250 & 0,0074 & 0,6029 & 0,3900 \\
\hline 0,4986 & 0,3801 & 0,1210 & 0,8440 & 0,1290 & 0,0270 & 0,0143 & 0,7235 & 0,2620 \\
\hline 0,5008 & 0,4002 & 0,0990 & 0,8064 & 0,1654 & 0,0280 & 0,0176 & 0,7720 & 0,2110 \\
\hline
\end{tabular}

As frações mássicas dos componentes foram encontradas através da inserção da densidade de cada fase encontrada no equilíbrio, nas curvas de calibração previamente construídas. A fração do terceiro componente foi determinada, por balanço de massa, sabendo que $\mathrm{w}_{1}+\mathrm{w}_{2}+\mathrm{w}_{3}=1$. As equações utilizadas são mostradas na Tabela 3.

Tabela 3 - Curvas de calibração para os sistemas Biodiesel de coco + metanol + água a $20{ }^{\circ} \mathrm{C} \mathrm{e} 60{ }^{\circ} \mathrm{C}$

\begin{tabular}{llll}
\hline & & Ajuste polinomial com sistemas a $20{ }^{\circ} \mathrm{C}$ & Ajuste polinomial para sistema a $60{ }^{\circ} \mathrm{C}$ \\
\hline Fase biodiesel & Biodiesel & $\rho=0,0073 \mathrm{x}^{2}+0,0700 \mathrm{x}+0,8001$ & $\rho=-0,0061 \mathrm{x}^{2}+0,0869 \mathrm{x}+0,7688$ \\
& Metanol & $\rho=0,0115 \mathrm{x}^{2}+0,0912 \mathrm{x}+0,8774$ & $\rho=0,0115 \mathrm{x}^{2}-0,0912 \mathrm{x}+0,8774$ \\
Fase água & Água & $\rho=-0,0657 \mathrm{x}^{2}+0,2637 \mathrm{x}+0,7992$ & $\rho=0,7576 \mathrm{x}^{2}-0,2935 \mathrm{x}+0,0687$ \\
& Metanol & $\rho=-0,0705 \mathrm{x}^{2}-0,1322 \mathrm{x}+0,9968$ & $\rho=0,9795 \mathrm{x}^{2}-0,1397 \mathrm{x}-0,0929$ \\
\hline
\end{tabular}

Observa-se para a fase rica em biodiesel uma baixa concentração de metanol. Este comportamento reflete a grande afinidade metanol/água, oriunda da presença de ligações hidrogênio, isto explica o álcool encontrar-se preferencialmente na fase rica em água. Segundo Rostami et al. (2012), os baixos valores da solubilidade de água no biodiesel, indicam que é necessária pouca energia para a separação por purificação.

A partir da análise da Figura 1, pode-se constatar uma pequena influência da temperatura na região de solubilidade dos sistemas. A curva binodal apresenta comportamento similar em ambas as temperaturas. Na fase rica em metanol, a $60{ }^{\circ} \mathrm{C}$, pode-se perceber uma maior solubilidade dos componentes, fato observado por uma curva levemente menor.

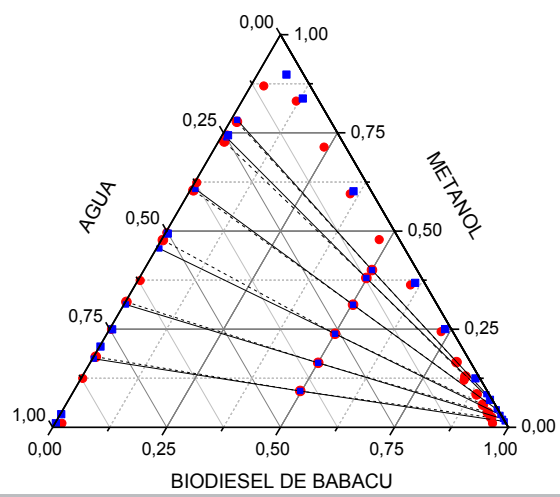


Figura 1 - Dados de equilíbrio líquido-líquido para o sistema biodiesel de babaçu + metanol + água: $20{ }^{\circ} \mathrm{C}$ $(\bullet), 60{ }^{\circ} \mathrm{C}(\square)$. Linhas de amarração: $20{ }^{\circ} \mathrm{C}(\bullet), 60{ }^{\circ} \mathrm{C}(\bullet)$.

Os perfis, da curva binodal e das linhas de amarração, são semelhantes aos apresentados por Mazutti et al. (2013) e Pinheiro et al. (2014), nos quais foram constatados a influência negligenciável da temperatura na região que delimita a zona homogênea e heterogênea, bem como uma baixa concentração de metanol e água na fase rica em biodiesel; e, alta concentração de metanol na fase rica em água.

\subsection{Coeficiente de Distribuição e de Seletividade}

Um importante parâmetro estudado na extração líquido-líquido é o coeficiente de distribuição $(\beta)$, que denota como o componente se distribui nas fases de estudo (Sandler, 2006).

O coeficiente de distribuição para o metanol (2) / água (3) foram calculados de acordo com as Equações 4 e 5.

$$
\begin{aligned}
& \beta_{2}=\frac{\text { Wmetanol }_{\text {fase rica em biodiesel }}}{\text { Wmetanol }_{\text {fase rica em água }}} \\
& \beta_{3}=\frac{\text { Wágua fase rica em biodiesel }}{\text { Wágua fase rica em água }}
\end{aligned}
$$

O parâmetro seletividade (S) que determina o potencial de extração do solvente é calculado a partir da Equação 6.

$$
\mathrm{S}=\frac{\beta_{3}}{\beta_{2}}
$$

A Tabela 4 apresenta os valores dos coeficientes de distribuição $(\beta)$ e seletividade $(\mathrm{S})$ para o sistema biodiesel de babaçu + metanol + água a $20^{\circ} \mathrm{C}$ e $60^{\circ} \mathrm{C}$.

Tabela 4 - Coeficientes de distribuição do metanol $\left(\beta_{1}\right)$, da água $\left(\beta_{2}\right)$ e a seletividade $(S)$ para o sistema

\begin{tabular}{|c|c|c|}
\hline$\beta_{1}$ & $\beta_{2}$ & $\mathrm{~S}$ \\
\hline$\left(\mathrm{w}_{1} \mathrm{I} / \mathrm{w}_{1}{ }^{\mathrm{II}}\right)$ & $\left(\mathrm{w}_{2}{ }^{\mathrm{I}} / \mathrm{w}_{2}{ }^{\mathrm{II}}\right)$ & $\left(\beta_{1} / \beta_{2}\right)$ \\
\hline \multicolumn{3}{|c|}{ biodiesel de babaçu + metanol + água a $60{ }^{\circ} \mathrm{C}$} \\
\hline 0,0286 & 0,1345 & 4,71 \\
\hline 0,0351 & 0,1149 & 3,28 \\
\hline 0,0471 & 0,1194 & 2,54 \\
\hline 0,0651 & 0,1382 & 2,12 \\
\hline 0,1029 & 0,1783 & 1,73 \\
\hline 0,1343 & 0,2142 & 1,59 \\
\hline
\end{tabular}
biodiesel de babaçu(1) + metanol (2) + água (3), a $20{ }^{\circ} \mathrm{C}$ e $60{ }^{\circ} \mathrm{C}$

\begin{tabular}{ccc}
\hline$\beta_{1}$ & $\beta_{2}$ & $\mathrm{~S}$ \\
\hline$\left(\mathrm{w}_{1}^{\mathrm{I}} / \mathrm{w}_{1}^{\mathrm{II}}\right)$ & $\left(\mathrm{w}_{2}{ }^{\mathrm{I}} / \mathrm{w}_{2}{ }^{\mathrm{II}}\right)$ & $\left(\beta_{1} / \beta_{2}\right)$ \\
\hline \multicolumn{2}{c}{ biodiesel de babaçu + metanol + água a $20{ }^{\circ} \mathrm{C}$} \\
\hline 0,0854 & 0,0003 & 298,64 \\
0,0688 & 0,0011 & 62,39 \\
0,0688 & 0,0028 & 24,40 \\
0,0750 & 0,0068 & 11,03 \\
0,0945 & 0,0178 & 5,30 \\
0,1073 & 0,0270 & 3,98 \\
\hline
\end{tabular}

Os baixos valores dos coeficientes de distribuição do metanol, obtidos para todos os sistemas em estudo, confirmam que este álcool se distribui preferencialmente na fase rica em água devido a presença do grupamento hidroxila. Os valores de seletividade, para todos os sistemas apresentaram valores superiores a 1 , confirmando também a boa capacidade extrativa por parte do solvente (água). 


\subsection{Confiabilidades dos Dados Experimentais}

A análise da confiabilidade termodinâmica dos dados experimentais de ELL foi avaliada pela correlação proposta por Othmer-Tobias (1942), conforme a Equação 7.

$$
\ln \left(\frac{1-w_{3}^{I I}}{w_{3}^{I I}}\right)=A+B *\left(\frac{1-w_{1}^{I}}{w_{1}^{I}}\right)
$$

onde $w_{3}^{I I}$ é a fração mássica de água na fase rica em água e $w_{1}^{I}$ representa a fração mássica de biodiesel na fase rica em biodiesel.

A Tabela 5 mostra as correlações dos dados experimentais deste trabalho. Os ajustes determinados para a correlação de Othmer-Tobias são próximos a unidade, o que resulta na confiabilidade dos dados experimentais.

Tabela 5 - Valores A, B e R² das correlações de Othmer-Tobias para o sistema em estudo

\begin{tabular}{cccc}
\hline & \multicolumn{3}{c}{ Correlação de Othmer-Tobias } \\
\cline { 3 - 4 } Temperatura & A & B & $\mathrm{R}^{2}$ \\
\hline & Biodiesel de babaçu + metanol + água & \\
\hline $20^{\circ} \mathrm{C}$ & 5,01 & 1,54 & 0,99 \\
$60^{\circ} \mathrm{C}$ & 3,97 & $-1,75$ & 0,97 \\
\hline
\end{tabular}

\subsection{Modelagem Termodinâmica: NRTL, UNIQUAC, UNIFAC}

As equações NRTL, UNIQUAC e UNIFAC foram utilizadas para correlacionar os dados experimentais dos sistemas de biodiesel de babaçu + metanol + água a $20^{\circ} \mathrm{C} \mathrm{e} 60{ }^{\circ} \mathrm{C}$. A Tabela 6 reporta os parâmetros de interação binária, determinados neste trabalho, para os modelos termodinâmicos NRTL e UNIQUAC. As Figuras 2 e 3 apresentam os gráficos comparativos entre os modelos termodinâmicos estudados e a Tabela 7 mostra matematicamente os desvios quadráticos médios para cada modelo quando relacionado aos dados experimentais.

Tabela 6 - Parâmetros de interação NRTL e UNIQUAC entre biodiesel (1)/ metanol (2) e água (3) a 20 ${ }^{\circ} \mathrm{C}$ e $60{ }^{\circ} \mathrm{C}$

\begin{tabular}{|c|c|c|c|c|c|c|c|c|}
\hline \multirow{3}{*}{ Par ij } & \multicolumn{8}{|c|}{ Modelo termodinâmica } \\
\hline & \multicolumn{3}{|c|}{ NRTL } & & \multicolumn{3}{|c|}{ UNIQUAC } & \multirow[b]{2}{*}{$B j i(1 / K)$} \\
\hline & $A i j$ & $A j i$ & $\operatorname{Bij}(1 / K)$ & $B j i(1 / K)$ & $A i j$ & $A j i$ & $\operatorname{Bij}(1 / K)$ & \\
\hline 14 & 38,179 & 1226,6 & 1,6980 & 0,93152 & 2871.6 & $-582,09$ & $-6,7110$ & 1,6485 \\
\hline 15 & 8510,5 & 3019,0 & 25,093 & 0,064941 & 7261,4 & 17,824 & $-20,139$ & 0,70328 \\
\hline 24 & 250,81 & 400,32 & 2,3131 & 3,1254 & 2060,2 & $-5914,4$ & $-7,2198$ & 21,055 \\
\hline
\end{tabular}

Através dos gráficos com os dados experimentais e modelados é possível verificar que para sistemas que apresentam moléculas hidrofóbicas, como água e álcool, erros importantes são encontrados para o modelo UNIFAC (Pinheiro et al., 2014). A principal razão para este desvio é o fato de que os parâmetros de interação para o modelo UNIFAC são determinados a partir de dados de equilíbrio líquido-vapor (em um intervalo de concentrações que podem 
dificultar a previsão da fase líquida à diluição infinita) para sistemas com álcool e água (Danner e Gupte,1986; Chen, 1993).

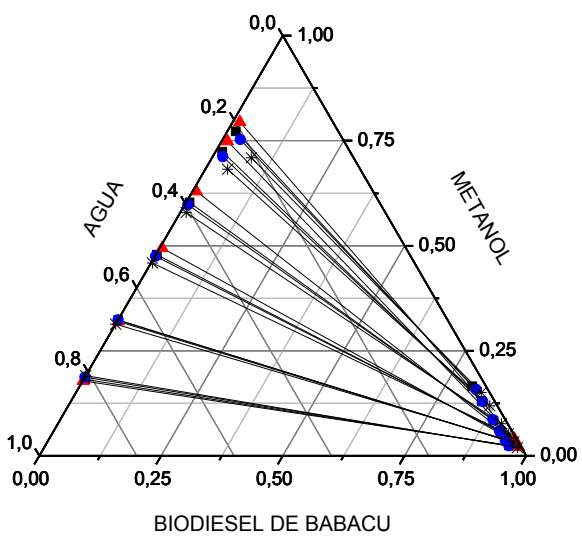

Figura 4 - Linhas de amarração experimentais (ם), calculadas pelo modelo NRTL ( $\boldsymbol{\Delta}$ ), UNIQUAC ( • ) e UNIFAC $(*)$ a $20^{\circ} \mathrm{C}$.

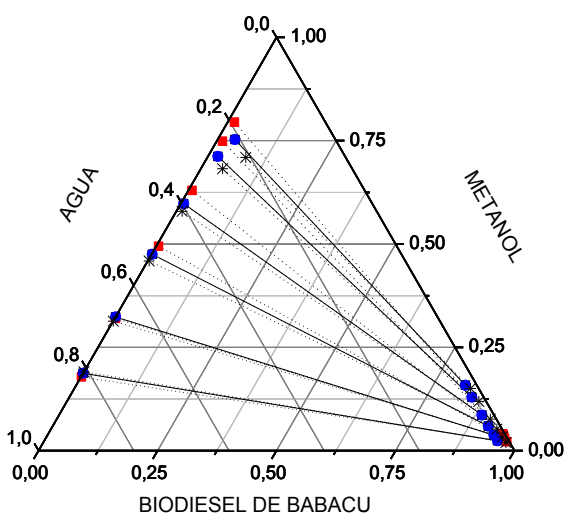

Figura 5 - Linhas de amarração experimentais (ם), calculada pelo modelo NRTL ( $\boldsymbol{\Delta}$ ), UNIQUAC (•) e UNIFAC $(*)$ a $60{ }^{\circ} \mathrm{C}$.

Tabela 7- Desvio médio quadrático para modelos termodinâmica para sistemas compostos de biodiesel de babaçu+ metanol+ água

\begin{tabular}{cccc}
\hline $\mathbf{T} /{ }^{\mathbf{C}}$ & NRTL & $\begin{array}{c}\text { Modelo termodinâmica } \\
\text { UNIQUAC }-\boldsymbol{\delta}_{\mathbf{x}}(\boldsymbol{\%})\end{array}$ & UNIFAC - $\boldsymbol{\delta}_{\mathbf{x}}(\boldsymbol{\%})$ \\
\hline Biodiesel de babaçu + metanol + água & & & \\
20 & 0,69 & 0,93 & 2,21 \\
60 & 1,04 & 0,65 & 5,15 \\
Desvio global médio & 0,88 & 0,80 & 3,96 \\
\hline
\end{tabular}

\section{CONCLUSÃO}

Com base nos dados experimentais de equilíbrio líquido-líquido dos sistemas estudados, foi verificada pouca influência da temperatura na solubilidade dos componentes. A correlação de Othmer-Tobias, apresentou coeficientes de regressão próximos da unidade, o que comprova a qualidade dos dados experimentais. Os coeficientes de distribuição apresentaram baixos valores indicando a distribuição preferencial do metanol na fase rica em água e os valores de seletividade obtidos, foram superiores a 1, comprovando ser a água um ótimo solvente para processo extrator estudado. Os dados foram correlacionados com os modelos NRTL, UNIQUAC e UNIFAC e apresentaram valores de desvio médio satisfatórios, que variaram entre $0,80 \%$ e $3,96 \%$.

\section{REFERÊNCIAS}

ABRAMS, D. S.; PRAUSNITZ, J. M. Statistical Thermodynamics of Liquid Mixtures: A New Expression for the Excess Gibbs Energy of Partly or Completely Miscible Substances, AIChE J., v. 21, p. 116-128, 1975.

CHEN F., HOLTEN- ANDERSEN J., TYLE H. New developments of the UNIFAC model for environmental application, Chemosphere, v. 26, p. 1325-1354, 1993. 
DANNER R. P.; GUPTE, P. A. Density dependent local composition models: an interpretive review. Fluid Phase Equilibr., v. 29, p. 415 - 430, 1986.

DE BONI, L. A. B; GOLDANI, E.; MILCHAREK, C. D.; SANTOS, F.A. Tratamento físicoquímico da água de lavagem proveniente da purificação de biodiesel. Tchê Química, v. 4, p.41-47, 2007.

FERRARI, R. A.; OLIVEIRA, V. S.; SCABIO, A. (a). Biodiesel de soja - Taxa de conversão em ésteres etílicos, caracterização físico-química e consumo em gerador de energia. Química Nova, v. 28, p. 19-23, 2005.

FREDENSLUND, A. A.; GMEHLING, J.; RASMUSSEN, P. Vapour-Liquid Equilibria using UNIFAC, Elsevier, Amsterdam, 1977.

KNOTHE, G.; VAN GERPEN, J.; KRAHL, J. Manual de biodiesel. 1. ed. São Paulo: Edgar Blücher, 2006.

LIMA, J.R.O.; SILVA, R.B.; SILVA, C.C.M.; SANTOS, L.S.S.; SANTOS JR, J. R.; MOURA, E. M.; MOURA, C. V. R. Biodiesel de babaçu (orbignya sp.) obtido por via etanólica. Química Nova, v. 30, p. 600 - 603, 2007.

MAGNUSSEN, T,; RASMUSSEN, P,; FREDENSLUND, A, A, UNIFAC Parameter Table for Prediction of Liquid-Liquid Equilibria, Ind, Eng, Chem, Process Des, Dev, v. 20, 331339, 1981,

MAZUTTI, M,A,; VOLL, F,A,P,; CARDOZO-FILHO, L,; CORAZZA, M,L,; LANZA, M,; PRIAMO, W,L,; OLIVEIRA, J,V, Thermophysical properties of biodiesel and related systems: (Liquid+liquid) equilibrium data for soybean biodiesel. J. Chem. Thermodyn. , v. 58, p. 83-94, 2013.

NELDER, J. A.; MEAD, R. A. A simplex method for function minimization. Comp. J., v.7, n. 4, p. 308-313, 1965

OTHMER, D. F.; TOBIAS, P. E. Tie line correlation. Ind. Eng. Chem., v. 34, n. 6, p. 693-696, 1942.

PINHEIRO, R. S; BESSA, A. M.M., QUEIROZ, B. A; DUARTE, A. M. S. F; SANT'ANA,H. B; SANTIAGO-AGUIAR, R.S. Optimization of the methylic biodiesel purification process byintermediate of liquid-liquid equilibrium data for ternary systems containing methanol + water + (soybean, corn or brown shell of coconut) biodiesel. Fluid phase Equilib., v 361, p. 30-36, 2014.

RENON, H; PRAUSNITZ, J. M. Local Compositions in Thermodynamics: Excess Functions for Liquid Mixtures, AIChE J., v. 14, p. 135-144, 1968.

ROSTAMI , M.; RAEISSI, S.; MAHMOUDI, M.; NOWROOZI, M. Liquid-Liquid Phase Equilibria of Systems of Palm and Soya Biodiesels: Experimental and Modeling Ind. Eng. Chem. Re, v. 51, p. 8302-8307, 2012.

SANDLER, S. I. Chemical, biochemical, and engineering thermodynamics. New York: John Wiley \& Sons Inc., 2006. 960 p.

STRAGEVITCH, L. Equilíbrio Líquido-Líquido em Sistemas Não Eletrolíticos. 1997. Tese (Doutorado em Engenharia Química) - Faculdade de Engenharia Química, Universidade Estadual de Campinas, Campinas, 1997.

STRAGEVITCH, L.; d'AVILA, S. G. Aplication of a Generalised Maxium Likelihood Method in the Reduction of Multicomponent Liquid-Liquid Equilibrium Data. Braz. J. of Chem. Eng., v. 14, p. 41-52, 1997.

ZHANG, Z.; HILL, G. A. Ternary Liquid-Liquid Equilibria of Water, Ethanol, and Oleic Acid. J. Chem. Eng. Data, v. 36, p. 453- 456, 1991. 